\title{
Uso de ferramentas de mineração para análise da absorção de água em formulações cerâmicas com resíduo oriundo da região da campanha
}

\author{
Use of mining tools to analyze water absorption in ceramic formulations with residue from the \\ campaign region \\ G. C. Mendonça ${ }^{1}$; C. C. Ferreira ${ }^{2 *}$; V. L. D. Ferreira ${ }^{3}$ \\ ${ }^{1}$ Acadêmica de Engenharia Química, Universidade Federal do Pampa, 96413-172, Bagé-RS, Brasil \\ ${ }^{2}$ Docente do Núcleo do Desenho Técnico, Universidade Federal do Pampa, 96413-172, Bagé-RS, Brasil \\ ${ }^{3}$ Docente da Licenciatura em Matemática, Universidade Federal do Pampa, 96413-172, Bagé-RS, Brasil
}

*cristiano.unipampa@gmail.com

\begin{abstract}
Os avanços tecnológicos têm como vantagens o barateamento de custos de componentes até mudanças de paradigmas, permitindo a coleta de grandes bases de dados para diversas aplicações. A utilização de ferramentas que permitam a captação, o gerenciamento e análise das informações, são fatores fundamentais a qualquer empresa que quer ter uma significativa participação no mercado. O presente trabalho tem como objetivo utilizar a mineração de dados para classificar corpos de prova cerâmicos formulados com diferentes percentuais de cinza de casca de arroz (0:0; 0:10; 0:20 e 0:30) por meio de ensaios de caracterização física (absorção de água). O processo de fabricação dos corpos de prova ocorreu por prensagem e a secagem foi realizada de modo natural e artificial, ao final desse processo os corpos de prova foram sinterizados na temperatura de $800^{\circ} \mathrm{C}$. A metodologia utilizada para caracterizar a absorção de água seguiu os procedimentos da M-CIENTEC 0-22 (1995). Para a aferição dos resultados encontrados no teste de absorção utilizou-se os parâmetros da norma NBR 15270-2005 e análise estatística através do software WEKA. Ao final observou-se que todos os corpos de prova da formulação 0:0 e 70\% da formulação 0:10 estão dentro dos índices físicos recomendados para blocos de vedação. As demais formulações apresentaram resultados fora do recomendado. Em relação ao software WEKA o algoritmo classificador escolhido para obter-se a árvore de decisão foi J48 e o Kappa statistic obtido foi de 0.88 .

Palavras-chave: cerâmica vermelha, absorção de água, minerção de dados.
\end{abstract}

Technological advances have the advantages of lowering costs of components to paradigm changes, allowing the collection of large databases for various applications. The use of tools that allow the capture, management and analysis of information are fundamental factors for any company that wants to have a significant market share. The present work aims to use data mining to classify ceramic test pieces formulated with different percentages of rice hull ash $(0: 0,0: 10,0: 20$ and $0: 30)$ by means of characterization tests (water absorption). The process of fabrication of the test specimens was done by pressing and the drying was performed in a natural and artificial way, at the end of this process the specimens were sintered at a temperature of $800^{\circ} \mathrm{C}$. The methodology used to characterize water absorption followed the procedures of M-CIENTEC 0-22 (1995). For the measurement of the results found in the absorption test, the parameters of the norm NBR 15270-2005 and statistical analysis through the WEKA software were used. At the end it was observed that all test bodies of the 0: 0 formulation and $70 \%$ of the 0:10 formulation are within the physical indexes recommended for sealing blocks. The other formulations presented results that were not recommended. In relation to the WEKA software the classifier algorithm chosen to obtain the decision tree was J48 and the statistic Kappa obtained was 0.88. Keywords: red ceramic, water absorption, data of mining.

\section{INTRODUÇÃO}

Atualmente, percebe-se entre os empresários do setor cerâmico que existem incertezas em relação ao potencial das matérias-primas disponíveis na região. Nesse sentido, o reaproveitamento de resíduos sólidos é muito importante para o meio ambiente, pois reduz o desperdício de recursos naturais, bem como minimiza os impactos ambientais em sua disposição final [1].

Segundo dados encontrados sobre o panorama da orizicultura Brasileira a maior região produtora de arroz, no Brasil, é o Rio Grande do Sul (RS) [2], e a casca de arroz é o subproduto gerado em 
maior quantidade no beneficiamento do grão, sendo uma matéria-prima de baixo custo e de difícil reaproveitamento por conter baixas propriedades nutritivas e possuir elevado teor de sílica [3].

De modo geral, as indústrias de cerâmica tradicional utilizam a argila como matéria-prima principal na fabricação de diversos materiais, tais como: blocos de vedação e estruturais, telhas e tijolos maciços [4]. Entre as diversas matérias primas provenientes da argila têm-se a do tipo caulinita que é composta por alumina e sílica, e quando submetida a altas temperaturas estes óxidos se combinam, influenciando nas propriedades mecânicas do material, como por exemplo a resistência e a absorção de água [5]. Em relação a absorção de água (AA) pode-se destacar que é uma propriedade física que está relacionada a porosidade do material, uma vez que, ao entrar em contato com a umidade, a água ocupa os espaços vazios na peça, preenchendo-os, outro fator de importante é que com o aumento da (AA) a resistência mecânica da peça diminui, tendo uma importância fundamental na qualidade final das peças cerâmicas [6].

O software WEKA (Waikato Enviroment for Knowledge Analysis) é uma ferramenta de mineração de dados em JAVA e se consolidou como uma das mais utilizadas em ambiente acadêmico [7]. Por meio de técnicas de Data Mining diversas empresas têm conquistado um diferencial frente aos seus concorrentes. Por exemplo, prevenção de tendências, aprimoramento de produtos ou serviços, e também aumentado seus lucros em face da grande competitividade entre comercial [8].

A utilização do WEKA no presente trabalho é importante para a classificação dos corpos de prova em relação ao parâmetro de absorção de água, sendo viável para a classificação em função do que preconiza a norma M-CIENTEC-0-22 (1995).

Nesse cenário, realizou-se nesta investigação uma análise de AA nos corpos de prova cerâmicos formulados com diferentes percentuais de CCA e a partir desses resultados desenvolveu-se o algoritmo por meio de mineração dados pelo software WEKA.

\section{MATERIAL E MÉTODOS}

Primeiramente foram coletadas a argila caulinita e a cinza de casca de arroz (CCA), ambas em uma olaria localizada na cidade de Bagé-RS. No segundo momento a argila foi levada para o Laboratório de Materiais da UNIPAMPA onde foi moída e peneirada até 100 mesh. A CCA foi apenas quarteada pois a sua granulometria se assemelhava com a da argila, além disso, deve-se destacar que a casca de arroz servia de combustível para os fornos da olaria que queimavam esses resíduos na temperatura de $700^{\circ} \mathrm{C}$ aproximadamente. Após, no terceiro momento, as amostras de argila in natura e resíduo nas proporções pré-determinadas foram misturadas e homogeneizadas. As formulações foram umidificadas até atingir $5 \%$ em peso (massa de umidade/massa seca) e foram preparadas conforme a Tabela 1 .

Tabela 1 - Formulações cerâmicas investigadas (\% em peso).

\begin{tabular}{ccc}
\hline Formulação & $\begin{array}{c}\text { Argila } \\
(\boldsymbol{\%})\end{array}$ & $\begin{array}{c}\text { CCA } \\
(\boldsymbol{\%})\end{array}$ \\
\hline A0 & 100 & 0 \\
CCA10 & 90 & 10 \\
CCA20 & 80 & 20 \\
CCA30 & 70 & 30 \\
\hline
\end{tabular}

Os corpos de prova foram moldados via prensagem, em uma prensa Atlas Series Laboratory Hydraulic Press, com 5 toneladas, foram submetidos a secagem natural, em temperatura ambiente por 24 horas e a secagem artificial em estufa modelo Fanem Orion 515 a $110^{\circ} \mathrm{C}$ por 24 horas. Em seguida, os corpos de prova foram queimados em forno elétrico SANCHIS, com um patamar de $80^{\circ} \mathrm{C}$ por hora e ao atingir a temperatura desejada $\left(800^{\circ} \mathrm{C}\right)$ os corpos de prova permaneceram no forno por mais 3 horas.

O ensaio de absorção de água seguiu os padrões da norma M-CIENTEC 0-22 (1995) onde primeiramente os corpos de prova foram fervidos por 2 horas e depois foram secos com um pano de algodão e pesados em uma balança eletrônica - Massa úmida $(\mathrm{Mu})$, por fim foram colocados na estufa a $110^{\circ} \mathrm{C}$ por 15 horas e pesados na balança eletrônica - Massa seca (Ms). A taxa de absorção de água foi calculada a partir da equação (1): 
absorção de água $=\frac{\mathrm{Mu}-\mathrm{Ms}}{\mathrm{Ms}} \times 100 \%$

Após, os resultados de AA foram inseridos no software WEKA versão 3.8 e a Tabela 2 mostra as tolerâncias ideias para que os produtos tenham condição de serem aplicados na fabricação de blocos de vedação.

Tabela 2 - Tolerâncias de absorção de água.

\begin{tabular}{|c|c|c|}
\hline Tolerância (\%) & Resposta & Simbologia \\
\hline$<8.00$ & Não atende & NA \\
\hline $8.00<\mathrm{AA}<10.99$ & Não atende & NA \\
\hline $11.00<\mathrm{AA}<17.99$ & Atende & A \\
\hline $18.00<\mathrm{AA}<22.00$ & Não atende & NA \\
\hline
\end{tabular}

No software WEKA, primeiramente selecionou-se a opção "WEKA Explorer que permite a inserção dos dados através de arquivo de extensão CSV data files (*.csv). Foram utilizados 4 atributos representados pelas questões encontradas na Tabela 2 conforme códigos. Outro item utilizado no software foi a aba "classify" que serve para realizar a tarefa de classificação, que consiste em aprender um modelo classificador no intuito de mapear cada conjunto de atributos para um dos rótulos de classe pré-determinados [9]. A "cross-validation" que usa validação cruzada do tipo K-fold e a "use training set" que usa os casos de treino como de teste. O algoritmo classificador escolhido para obter-se a árvore de decisão foi J48, por ser bastante adequado na mineração de dados com variáveis qualitativas contínuas e discreta [10].

\section{RESULTADOS E DISCUSSÃO}

A Tabela 3 mostra o percentual de absorção de água em cada corpo de prova, assim como a classificação de acordo com a Tabela 2. A NBR 15270-2005 indica que para blocos cerâmicos para alvenaria estrutural a AA deve estar entre 8 e $22 \%$, mostrando que todas as formulações (0:0) estão dentro do recomendado pela norma, de acordo com a Tabela 3. A formulação (0:10) apresentou aproximadamente $72 \%$ das amostras como dentro do recomendado. As demais formulações $(0: 20$ e 0:30) apresentaram todas as amostras classificadas como fora do recomendado.

A classificação realizada através da Tabela 2 e 3 dos corpos de prova foi plotada no software WEKA que, por sua vez, gerou as imagens da Figura 1. Nessa figura estão representadas as quatro formulações (A0, CA10, CA20 e CA30), respectivamente. A classificação utilizando o software WEKA mostrou que $44 \%$ dos corpos de prova atenderam ao que foi especificado na Tabela 2.

Tabela 3 - Resultado da absorção de água e classificação.

\begin{tabular}{|c|c|c|c|c|c|c|c|c|}
\hline CP & $\begin{array}{l}\text { AA } \\
\text { 0:0 }\end{array}$ & $\begin{array}{c}\text { Tolerância } \\
\text { 0:0 }\end{array}$ & $\begin{array}{c}\text { AA } \\
0: 10\end{array}$ & $\begin{array}{c}\text { Tolerância } \\
\text { 0:10 }\end{array}$ & $\begin{array}{c}\text { AA } \\
0: 20\end{array}$ & $\begin{array}{c}\text { Tolerância } \\
\text { 0:20 }\end{array}$ & $\begin{array}{l}\text { AA } \\
\mathbf{0 : 3 0}\end{array}$ & $\begin{array}{c}\text { Tolerância } \\
\mathbf{0 : 3 0}\end{array}$ \\
\hline 1 & 13.9 & $\mathrm{~A}$ & 18.1 & NA & 19.5 & NA & 21.1 & NA \\
\hline 2 & 13.5 & A & 18.2 & NA & 20.9 & NA & 21.7 & NA \\
\hline 3 & 14.6 & A & 18.0 & NA & 20.1 & NA & 21.7 & NA \\
\hline 4 & 13.4 & A & 17.5 & A & 20.7 & NA & 21.0 & NA \\
\hline 5 & 15.8 & A & 16.5 & A & 20.0 & NA & 21.9 & NA \\
\hline 6 & 15.0 & A & 16.5 & A & 20.6 & NA & 21.6 & NA \\
\hline 7 & 14.7 & A & 17.4 & A & 20.9 & NA & 20.8 & NA \\
\hline 8 & 14.0 & A & 17.0 & A & 20.6 & NA & 20.1 & NA \\
\hline 9 & 14.0 & A & 16.8 & A & 19.6 & NA & 20.0 & NA \\
\hline 10 & 13.6 & A & 17.5 & A & 20.7 & NA & 21.5 & NA \\
\hline 11 & 13.2 & A & 17.6 & A & 19.0 & NA & 21.7 & NA \\
\hline 12 & 15.0 & A & 17.1 & A & 19.5 & NA & 20.8 & NA \\
\hline
\end{tabular}




\begin{tabular}{|c|c|c|c|c|c|c|c|c|}
\hline 13 & 13.4 & A & 18.5 & NA & 20.1 & NA & 20.1 & NA \\
\hline 14 & 14.7 & A & 18.6 & NA & 18.9 & NA & 21.9 & NA \\
\hline 15 & 14.1 & A & 17.6 & A & 18.2 & NA & 20.0 & NA \\
\hline 16 & 13.6 & A & 17.0 & A & 19.2 & NA & 20.7 & NA \\
\hline 17 & 14.1 & A & 16.9 & A & 19.5 & NA & 19.6 & NA \\
\hline 18 & 13.4 & A & 12.1 & A & 19.3 & NA & 20.4 & NA \\
\hline 19 & 13.3 & A & 20.5 & NA & 19.2 & NA & 19.8 & NA \\
\hline 20 & 13.7 & A & 16.7 & $\mathrm{~A}$ & 18.9 & NA & 22.2 & NA \\
\hline 21 & 13.4 & A & 18.2 & NA & 19.6 & NA & 19.6 & NA \\
\hline 22 & 13.8 & A & 17.5 & A & 19.3 & NA & 21.6 & NA \\
\hline 23 & 13.8 & A & 17.5 & A & 19.5 & NA & 20.7 & NA \\
\hline 24 & 14.6 & A & 16.9 & A & 18.9 & NA & 20.9 & NA \\
\hline 25 & 13.6 & A & 16.9 & A & 19.5 & NA & 20.7 & NA \\
\hline
\end{tabular}

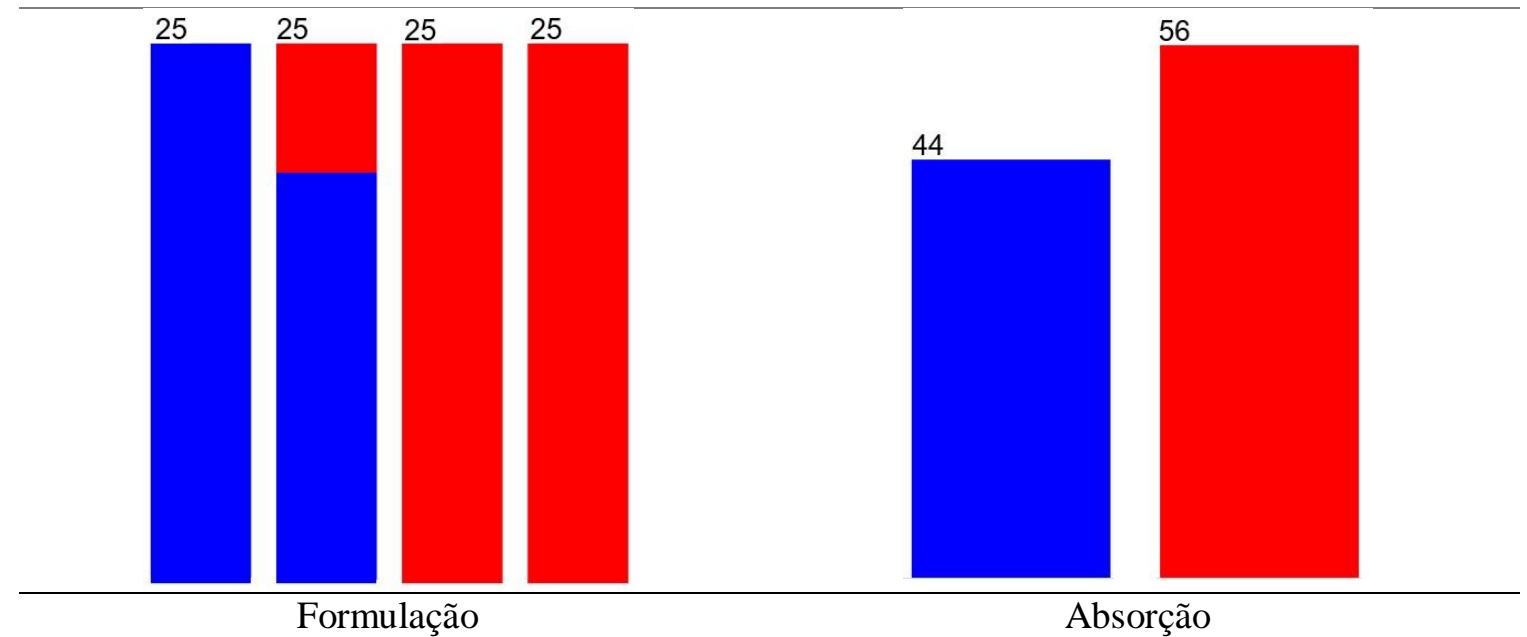

Figura 1 - Classificação das formulações no WEKA. Azul: formulações que estão dentro da tolerância, Vermelho: formulações que estão fora da tolerância.

A Tabela 4 apresenta o resultado da classificação usando o classificador J48 unpruned tree do modelo full training set.

Tabela 4 - Sumário da execução para o modo de teste selecionado.

\begin{tabular}{ccc}
\hline Instâncias e atributos & $\begin{array}{c}\text { Evaluate on training data } \\
\text { (unpruned) }\end{array}$ \\
\hline Correctly classified instances & $94 \%$ & 94 \\
Incorrectly classified instances & $6 \%$ & 6 \\
Kappa statistic & 0.88 & - \\
Mean absolute error & 0.0912 & - \\
Root mean squared error & 0.2135 & - \\
Relative absolute error & $18.5012 \%$ & - \\
Root relative squared error & $43.0191 \%$ & - \\
Total number of instances & 100 & - \\
\hline
\end{tabular}


Na Tabela 4 foi apresentado o parâmetro kappa statistic obtido para AA de 0,88. Esse índice é usado como uma medida de associação para descrever e testar o grau de concordância (confiabilidade e precisão) na classificação [11]. Os valores de Kappa acima 0,75 são caracterizados como de excelente concordância [12].

\section{CONCLUSÃO}

Conclui-se de acordo com a NBR 15270-2005 que todas as formulações estão dentro do padrão estabelecido. Ao analisar o parâmetro de AA para blocos de vedação no software WEKA apenas 44\% dos corpos de prova atenderam a especificação de absorção de água entre 11.00 e $17.99 \%$. O coeficiente Kappa statistic obtido foi de 0.88 o que demonstra o alto grau de confiabilidade e precisão.

\section{REFERÊNCIAS BIBLIOGRÁFICAS}

1. Da Silva EJ, Tashima MM, Akasaki JL. Estudo de reações expansivas em argamassas de cimento Portland com cinza de casca de arroz (CCA). Holos Environm. 2006 Jun;7(1):72-86, doi: 10.14295/holos.v7i1.974.

2. Zanin V. Panorama geral da orizicultura brasileira. Indicadores Econômicos FEE. 2013 Jul;41(2):51-66.

3. Garcia E, Cabral JM, Quarcioni VA, Chotoli FF. Avaliação da atividade pozolânica dos resíduos de cerâmica vermelha produzidos nos principais polos ceramistas do Estado de São Paulo. Cerâmica. 2015 Abr;61(358):251-258, doi: 10.1590/0366-69132015613581847.

4. Figueiredo JMR, Fernandes IMM, Silva VJ, Neves GA, Ferreira HC, Santana LNL. Influência da composição e das variáveis de processamento deformulações à base de argilas-uso em materiais refratários. Cerâmica. 2018 Mar;64(369):10-19, doi: 10.1590/0366-69132018643692169.

5. Fernandes IJ, dos Santos EC, Oliveira R, Reis JM, Calheiro D, Moraes CA, Modolo RC. Caracterização do resíduo industrial casca de arroz com vistas a sua utilização como biomassa. In Forum Internacional de Resíduos Sólidos-Anais. 2017 Ago;6(6):9.

6. Zaccaron A, Galatto SL, de Souza NV, Fernandes P. Avaliação da resistência mecânica e absorção de água em cerâmica vermelha com incorporação de chamote. Scientia Plena. 2018 Fev;14(2):1-8, doi: 10.14808/sci.plena.2018.029901.

7. Brooks CA, Thompson C, Kovanović V. Introduction to data mining for educational researchers. In Proceedings of the Sixth International Conference on Learning Analytics \& Knowledge. 2016 Apr;1(5):505-506. doi10.1145/2883851.2883879.

8. Gomes JCM, de Morais PH, Maia CM, Rodrigues WM. Técnicas de Mineração de Dados aplicado na Universidade Federal Rural do Semi-Árido (UFERSA) Campus Angicos. Anais do Encontro de Computação do Oeste Potiguar ECOP/UFERSA. 2017;1(1):31-35.

9. Tan PN, Steinbach M, Kumar V. Introdução ao datamining: mineração de dados. Ciência Moderna; 2009. $928 \mathrm{p}$.

10. Librelotto SR, Mozzaquatro PM. Análise dos algoritmos de mineração J48 e Apriori aplicados na detecção de indicadores da qualidade de vida e saúde. Rev Interdiscip Ens, Pesqui Extens. 2013;1(1):26-37.

11. Kotz S, Johnson NL. Encyclopedia of statistical sciences. New York: John Wiley \& Sons. 1983; 352 p.

12 Landis JR, Koch GG. The measurement of observer agreement for categorical data. Biometrics. 1977;33(1):159-174. 